\title{
The Relay of High-Frequency Sensory Signals in the Whisker-to-Barreloid Pathway
}

\author{
Martin Deschênes, Elena Timofeeva, and Philippe Lavallée \\ Centre de Recherche Université Laval-Robert Giffard, Hôpital Robert-Giffard, Quebec City, Quebec G1J 2G3, Canada
}

The present study investigated the operational features of whisker-evoked EPSPs in barreloid cells and the ability of the whisker-tobarreloid pathway to relay high rates of whisker deflection in lightly anesthetized rats. Results show that lemniscal EPSPs are single-fiber events with fast rise times $(<500 \mu \mathrm{sec})$ that strongly depress at short inter-EPSP intervals. They occur at short latencies $(3.84 \pm 0.96$ $\mathrm{msec})$ with little jitters $(<300 \mu \mathrm{sec})$ after electrical stimulation of the whisker follicle. Waveform analysis indicates that one to three lemniscal axons converge on individual barreloid cells to produce EPSPs of similar rise times but different amplitudes. When challenged by high rates of whisker deflection, cells in the whisker-to-barreloid pathway demonstrate a remarkable frequency-following ability. Primary vibrissa afferents could follow in a phase-locked manner trains of sinusoidal deflections at up to $1 \mathrm{kHz}$. Although trigeminothalamic cells could still faithfully follow deflection rates of $200-300 \mathrm{~Hz}$, the actual frequency-following ability of individual cells depends on the amplitude, velocity, and direction of displacements. The discharges of trigeminothalamic cells induce corresponding phase-locked EPSPs in barreloid cells, which trigger burst discharges at stimulus onset. During the following cycles of the stimulus train, few action potentials ensue because of the strong synaptic depression at lemniscal synapses. It is concluded that the whisker-to-barreloid pathway can relay vibratory inputs with a high degree of temporal precision, but that the relay of this information to the cerebral cortex requires the action of modulators, and possibly phase-locked discharges among an ensemble of relay cells.

Key words: trigeminal sensory nucleus; ventral posterior medial nucleus; thalamic relay cells; vibrissa; medial lemniscus; vibrissa primary afferents; barrel

\section{Introduction}

Behavioral studies have demonstrated that rats can use their whiskers to discriminate fine-grained textured surfaces (GuicRobles et al., 1989; Carvell and Simons 1990). Presumably, this ability requires the whisker-barrel system to be highly sensitive to rapid, small changes in the position of vibrissal hairs as they move across the surface of the object. Neurophysiological studies have shown that trigeminal primary afferents are exquisitely sensitive to small whisker deflections (Zucker and Welker, 1969; Gibson and Welker, 1983a,b; Lichtenstein et al., 1990; Shoykhet et al., 2000) and can encode in a one-to-one manner highfrequency vibrations (up to $1.5 \mathrm{kHz}$ ) (Gottschaldt and VahleHinz, 1981). However, little information is currently available on the frequency-following ability of cells in the relay stations of the whisker-to-barrel pathway. Although periodic whisker stimulation has been used in some studies of central neurons (Shipley, 1974; Hartings and Simons, 1998; Ahissar et al., 2000; Sosnik et al., 2001; Castro-Alamancos, 2002b), the range of frequencies used was low (usually $<40 \mathrm{~Hz}$ ) in comparison with the frequency-following ability of peripheral afferents.

A number of studies suggest that the vibrissa system of rodents is designed to respond robustly and rapidly to changes in sensory

\footnotetext{
Received March 14, 2003; revised June 6, 2003; accepted June 10, 2003.

This work was supported by Canadian Institutes for Health Research Grant MT-5877 to M.D. and a Natural Sciences and Engineering Research Council postdoctoral fellowship to E.T.

Correspondence should be addressed to Dr. Martin Deschênes, Centre de Recherche Université Laval-Robert Giffard, 2601 de la Canardière, Quebec City, Quebec G1J 2G3, Canada. E-mail: martind@globetrotter.net. Copyright $\odot 2003$ Society for Neuroscience $\quad$ 0270-6474/03/236778-10\$15.00/0
}

input (Shoykhet et al., 2000; Miller et al., 2001; Pinto et al., 2003). Among the supporting evidence is the fast time course and unitary character of the EPSPs evoked in thalamic cells of the ventral posterior medial nucleus (VPM) by medial lemniscus or sensory stimulation (Brecht and Sakmann, 2002; Castro-Alamancos, 2002a,b). Whether high-frequency whisker deflections elicit corresponding patterns of EPSPs in thalamic cells will obviously depend on the frequency-following ability of cells in the principal trigeminal nucleus (PR5) and the reliability of transmission in the whisker-to-barreloid disynaptic pathway. Factors such as synaptic depression, the degree of axonal convergence, and local inhibitory actions are also expected to shape the frequency response profiles at the PR5 and VPM levels. In the present study, we addressed two related issues: first, we analyzed the waveform of EPSPs evoked in VPM cells by sensory and electrical stimulation, and from that analysis, we attempted to estimate the number of lemniscal fibers that impact on single relay cells; second, we examined the responses of PR5 and VPM neurons to highfrequency whisker deflections. Results show that, although the frequency-following ability of PR5 cells depends on the amplitude, velocity, and direction of whisker motion, their discharges and the related patterns of EPSPs induced in VPM cells can follow in a one-to-one, phase-locked manner whisker deflections at hundreds of cycles per second.

\section{Materials and Methods}

Animal preparation. Experiments were performed in 42 adult rats (Sprague Dawley; 250-300 gm) in accordance with federally prescribed animal care and use guidelines. Under ketamine ( $75 \mathrm{mg} / \mathrm{kg})$-xylazine (5 

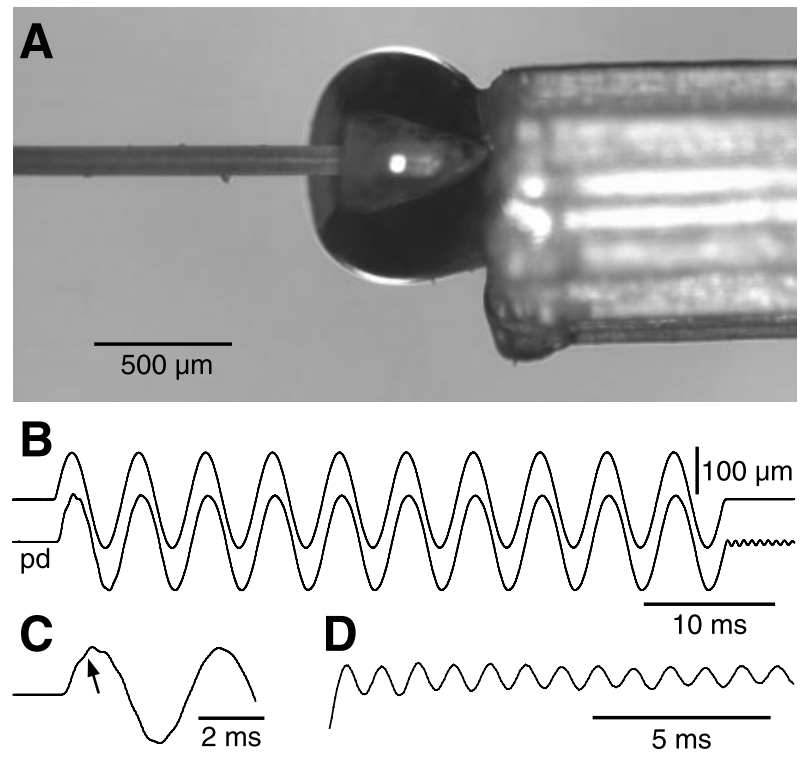

Figure 1. Mechanical properties of the piezoelectric stimulator used for high-frequency whisker deflection. $A$, The probe consists of a straw attached to the bimorph bender. The cut tip of the vibrissa was inserted in the cone-shaped glass bead glued in the free end of the straw. $B$, The frequency-following property of the probe was monitored with a photodiode (pd) (deflection amplitude, $100 \mu \mathrm{m}$; stimulus frequency, $175 \mathrm{~Hz}$ ). Note the notch (C, arrow) at stimulus onset and the ringing at stimulus offset $(D)$ that reflect the resonance frequency of the probe $(\sim 1 \mathrm{kHz})$.

$\mathrm{mg} / \mathrm{kg}$ ) anesthesia, the left facial nerve was cut, and the rat was placed in a stereotaxic apparatus. Throughout the experiment, the animal breathed freely, and body temperature was maintained at $37.5^{\circ} \mathrm{C}$ with a thermostatically controlled heating pad. Two stainless-steel tubes (diameter, $1.5 \mathrm{~mm}$; length, $15 \mathrm{~mm}$; spacing, $10 \mathrm{~mm}$ ) were fixed across the surface of the skull by means of screws and acrylic cement. Trephine holes were drilled, and ear bars were removed. For the recording session, the rat's head was maintained in a stereotaxic position by means of a small U-shaped frame bearing adjustable pins inserted in the tube openings of the cemented device. The frame was secured to a large steel post so that whiskers on the left mystacial pad were freely accessible for stimulation. In five experiments, a coaxial stimulating electrode was also placed in the medial lemniscus (frontal plane, $6.5 \mathrm{~mm}$ behind the bregma; lateral plane, $1 \mathrm{~mm}$ from the midline) (Paxinos and Watson, 1986). Before the start of recordings, the nape of the neck was infiltrated with longlasting local anesthetics (1\% Marcaine) to reduce animal discomfort. Unexpectedly, local anesthesia produced a remarkably still preparation in which the electroencephalogram (recorded in two rats) displayed spindles and a dominance of $5-7 \mathrm{~Hz}$ activity. Animals remained motionless with occasional twitches of the right whiskers, indicating that they did not experience any discomfort, but they briskly reacted to a moderate pinch of the hindlimbs. Together, these signs are indicative of a light anesthesia stage (stage III-2) (Friedberg et al., 1999). An additional dose of anesthetics (one-third of the initial dose) was usually given before inserting stimulating electrodes in the whisker pad (see below) or when small amplitude whisking movements were noticed.

Whisker stimulation. A whisker stimulator was built with a ceramic bimorph bender (Physik Instrumente, Karlsruhe, Germany) to which a thin straw was attached with cyanoacrylate glue. The free end of the straw contained a tiny, cone-shaped, glass bead in which the tip of the whisker could be snuggly inserted without any dead space (Fig. $1 A$ ). The beadstraw probe weighed $\sim 4 \mathrm{mg}$. The piezo amplifier (Physik Instrumente) was driven by bandpass-filtered $(1-500 \mathrm{~Hz})$ sinusoidal or triangular waveforms (Agilent Technologies, Palo Alto, CA). To prevent ringing of the stimulator at low frequency, the bimorph was blocked at midlength, which reduced maximal probe displacement to $\pm 140 \mu \mathrm{m}$. The reduction in displacement was compensated by deflecting the vibrissa at $5 \mathrm{~mm}$ from the pad. In test experiments conducted with a photodiode, this stimula- tor displayed the following characteristics to sinusoidal deflections of 100 $\mu \mathrm{m}$ : response time lag, $300 \mu \mathrm{sec}$; resonance frequency, $\sim 1 \mathrm{kHz}$ (Fig. $1 B-D)$. Deflection amplitudes were measured under a microscope equipped with a digital camera operated in the integration mode. The width of the shadow produced by sinusoidal displacements at various frequencies was compared with that produced by steady displacements. Virtually no attenuation was observed for deflection amplitudes of $<100$ $\mu \mathrm{m}$ at frequencies up to $200 \mathrm{~Hz}$. Beyond this frequency, filtering of the driving voltage introduced progressive attenuation of the deflection amplitude.

Data collection and analysis. Extracellular and/or intracellular recordings were obtained from VPM and PR5 neurons and from primary afferent axons in the vicinity of the PR5 nucleus. Cells were recorded using glass micropipettes (tip diameter, $\sim 0.5 \mu \mathrm{m}$; DC resistance, $30-40 \mathrm{~m} \Omega$ ) filled with a K-acetate solution $(0.5 \mathrm{M})$. Once an intracellular recording had been established, EPSPs evoked by manual deflection of individual whiskers were sampled over a $2-10 \mathrm{~min}$ period. Then the piezoelectric stimulator was positioned to deflect the principal whisker in different directions with bursts of 10 cycle stimuli. Most VPM and PR5 cells strongly responded to one whisker and more weakly to one to three adjacent whiskers, whereas other cells robustly reacted to the motion of four to eight whiskers; latter units were classified as multiwhisker cells. After the tests, electrical stimulation of the whisker follicle was attempted by inserting two tungsten microelectrodes on each side of the follicle to determine conduction velocity in the pathway.

Signals were amplified and low-pass-filtered at $3 \mathrm{kHz}$ by conventional means. Analog signals were digitized at $20 \mathrm{kHz}$ (Powerlab; AD Instruments, Castle Hill, Australia), stored on hard disks, and analyzed off-line using commercially available software (Chart 4.0; AD Instruments; Excel; Microsoft, Redmond, WA). Peristimulus-time histograms (PSTHs) of 10-20 responses were compiled in bin width of $200 \mu \mathrm{sec}$. When conduction times between the pad and recording sites could be determined by electrical stimulation, this delay plus the response time lag of the stimulator were subtracted from the records and PSTHs to better estimate the stimulus-response phase relationship.

\section{Results}

\section{Database}

This report is based on the intracellular recording of 51 whiskerresponsive VPM cells. Analysis of sensory-evoked EPSPs was performed in 33 cells that maintained stable resting membrane potentials (more negative than $-55 \mathrm{mV}$ ) for periods of $10-120 \mathrm{~min}$. When required, hyperpolarizing currents $(<2 \mathrm{nA})$ were injected into the cells to prevent spike discharges. Extracellular recordings were also obtained from 32 primary afferent axons and 28 PR5 units to study their ability to encode and relay high rates of whisker deflection. Primary afferent axons were distinguished from PR5 units by their strictly positive action potentials and their capacity to follow electrical stimulation of the follicles at high frequencies (e.g., 400-600 Hz).

\section{Properties of whisker-evoked lemniscal EPSPs}

The most striking feature of EPSPs evoked by whisker deflections in barreloid cells is their all-or-none unitary character (Fig. 2). Figure 2, $H$ and $I$, shows representative responses evoked in two barreloid cells by displacements of increasing amplitude $(H)$ and by suprathreshold deflections in different directions $(I)$. One can note that EPSPs occur in an all-or-none manner at threshold amplitude, and that increasing the amplitude or velocity of sinusoidal displacements, or changing their direction, never induces smooth, graded increases in synaptic responses. Instead, changes in stimulus parameters modify the latency and/or the number of unitary events. Responses evoked by medial lemniscus or follicular stimulation exhibit the same unitary character (Fig. $2 D$; see also $4 D)$. At low stimulus rate $(\sim 1 \mathrm{~Hz})$, they occur as discrete all-or-none events of fixed amplitude. Thus, like retinal EPSPs in 
the lateral geniculate nucleus (Turner and Salt, 1998) and lemniscal EPSPs in the ventral posterior lateral nucleus (Pinault and Deschênes, 1992), whisker-evoked EPSPs in barreloid cells present all of the characteristics of single-fiber events.

Whereas EPSPs evoked by whisker deflection display fast rise times (see below), their falling phase is prolonged and most often composed of two phases: an early fast decay followed by a slower phase that could last up to $40 \mathrm{msec}$ (Fig. $2 A-D$ ). As a rule, the second component is absent when EPSPs superimpose onto an IPSP (Fig. 2C) and is less prominent in cells recorded after ketamine injections. These observations are consistent with the possibility that the late component is generated, at least in part, by the activation of NMDA receptors (Salt, 1987). As previously reported (Castro-Alamancos, 2002a,b), EPSPs evoked by medial lemniscus or whisker stimulation display synaptic depression, the depression being especially important within sequences composed of three to four EPSPs occurring at intervals shorter than 100 msec (Figs. $2 E$ and $4 A$ ).

The bisynaptic pathway that links the follicles to the barreloids is fast conducting and highly secure. After electrical stimulation of single follicles, EPSPs occur at a mean latency of $3.84 \pm 0.96 \mathrm{msec}$ (Fig. $2 G$ ), and in any individual cell, EPSP jitters are typically $<300 \mu$ sec for suprathreshold stimuli $(F)$.

\section{Rise times of whisker-evoked EPSPs}

Figure $3 A$ shows representative scatter plots of the amplitudes versus rise times of EPSPs evoked by whisker deflection in three barreloid cells. Measurements were made for nonoverlapping EPSPs, and rise times were measured between 10 and $90 \%$ of peak amplitude. One can note that, despite large variations in amplitude, all of the rise times are uniformly fast and little dispersed in individual cells. The histogram of Figure $3 B$ shows the distribution of EPSP rise times across a population of 33 barreloid cells, and $C$ shows the SDs around the means for individual cells. Uniform rise times characterize not only EPSPs evoked in individual cells by principal whisker deflections but also those evoked in multiwhisker units by separate deflection of several vibrissas. The series of scatter diagrams of Figure $3 D$ is representative of similar results obtained in five multiwhisker cells. After deflection of each whisker composing the receptive field of this unit, all of the EPSPs displayed similar rise times, including EPSPs of much smaller amplitude that revealed a distinct set of synaptic contacts on this cell (Fig. 3D, inset within plot labeled C1). This narrow range of rise times indicates that lemniscal fibers that converge on a cell contact dendrites at equivalent electronic distance from the soma. However, the large variation in EPSP amplitude cannot be solely attributed to a mechanism of synaptic depression, because sudden jumps in amplitude were commonly observed at short inter-EPSP intervals.

\section{Convergence of lemniscal axons on barreloid cells}

Stimulation of the medial lemniscus in slices was reported to evoke a single unitary EPSP in VPM cells, even when stimulus intensity was increased well above threshold (Castro-Alamancos, 2002a). Although the number of lemniscal axons with intact connections is likely reduced in slices, this result nevertheless suggests that single barreloid cells receive contacts from few lemniscal fibers. In some cells, distinct populations of EPSPs could be clearly distinguished by their large amplitude difference, but in most cells, synaptic depression may have obscured size differences. To more precisely estimate the number of lemniscal axons that impact on single relay cells, we carefully inspected all of our records to find evidence for convergent connections. Our analysis relies on the assumption that all of the lemniscal synapses have a high release probability and depress upon repetitive activation (Castro-Alamancos, 2002a). If the second of two EPSPs occurring within a time interval of $75 \mathrm{msec}$ overshot the amplitude of the first by $25 \%$ after subtraction, it was concluded that the second EPSP resulted from the firing of a different presynaptic axon 

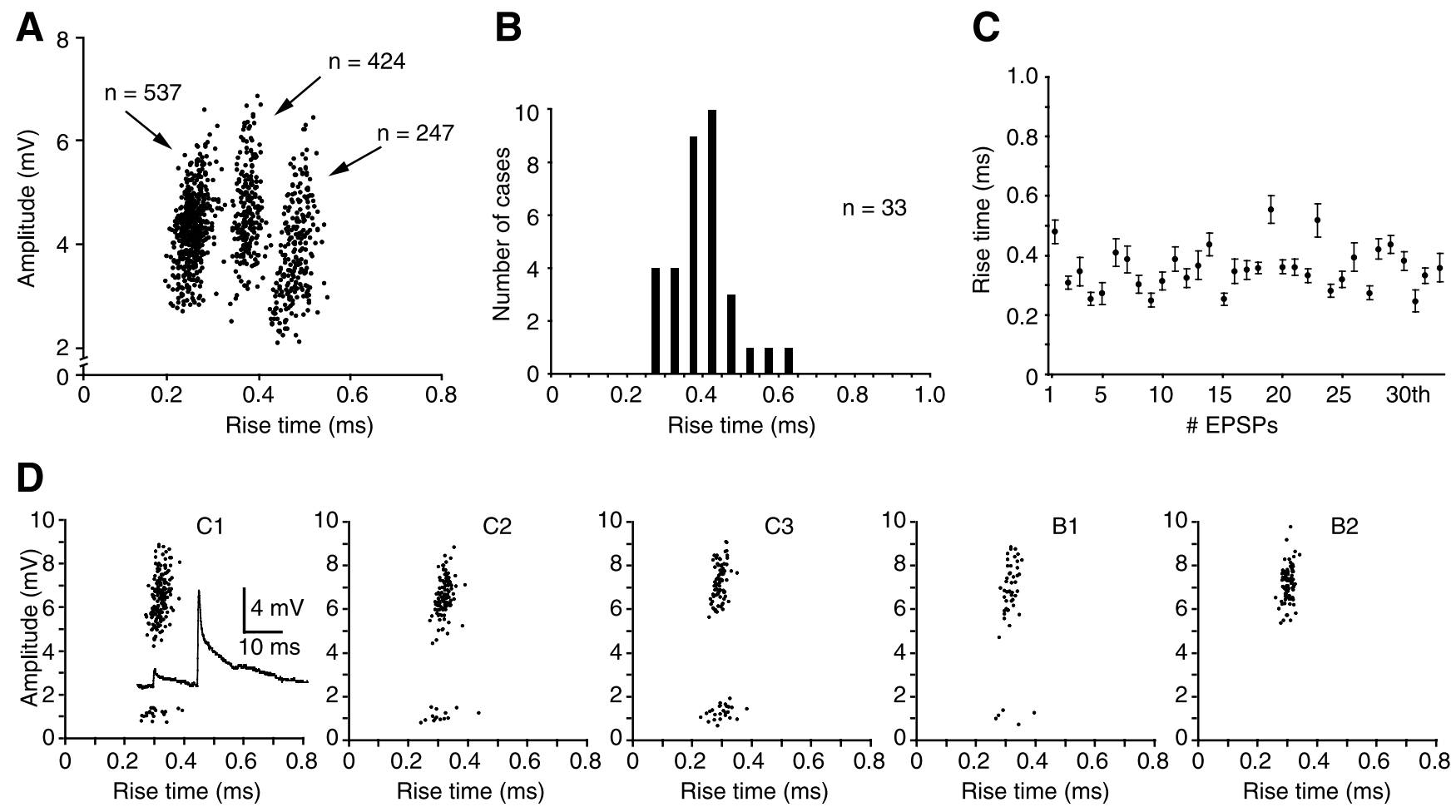

Figure 3. Amplitude and rise time characteristics of whisker-evoked EPSPs in barreloid cells. $A$, Scatter plots show the relationship between the amplitudes and rise times (10-90\% of peak amplitude) of EPSPs evoked by principal whisker deflection in three barreloid cells. The number of EPSPs in each cluster is indicated by arrows. $B$, The distribution of rise times across a population of 33 cells is shown. C, Graph shows the small dispersion of rise time values (error bars, SD). D, Plots show the relationship between the amplitudes and rise times of EPSPs evoked in a multiwhisker cell by deflecting separately each whisker composing its receptive field (whiskers are identified above the plots). Note the presence of at least two EPSPs of different amplitude (inset in plot C1), and their similar rise time values.

(Fig. $4 B, C$ ). Ten sequences of overshooting were required to confirm that conclusion in any individual cell. A time interval of $75 \mathrm{msec}$ was chosen, because depression was reported to be particularly strong above $10 \mathrm{~Hz}(>30 \%)$ (Castro-Alamancos, 2002a), and a $25 \%$ overshoot appeared as a criterion stringent enough to avoid questionable conclusions. On the basis of this analysis, it was found that barreloid cells receive input from one to three fibers (Fig. $4 F$ ). For $60 \%$ of the cells in which two lemniscal inputs were detected, our analysis was supported by evoking two populations of unitary EPSPs of different amplitude after lemniscal or follicular stimulation (Fig. 4D,E).

\section{Frequency-following ability in the} whisker-to-barreloid pathway

The above results show that individual barreloid cells receive input from one to three lemniscal axons, and that the relay of information between the follicles and barreloids is fast and secure. These features are expected to confer on the vibrissa system fine-grained resolution in the spatial and temporal domains. Thus, in additional experiments, we investigated how highfrequency whisker deflections are relayed through each component of the subcortical pathway.

The capacity of primary vibrissa afferents to follow high rates of sinusoidal deflections has been reported previously in rats and cats (Gottschaldt and Vahle-Hinz, 1981; Gibson and Welker, 1983b). We reexamined this issue to obtain a comparative basis for the responsiveness of PR5 and barreloid cells under similar conditions of stimulation. In these experiments, whiskers were deflected with amplitudes that produced suprathreshold responses at frequencies of $\geq 10 \mathrm{~Hz}$. When challenged by increasing rates of deflection, both tonic and phasic primary afferents dis- charged action potentials that remained phase locked to each cycle of the stimulus over a frequency range that far exceeded the compliance of the stimulator (Fig. 5). In units sensitive to small displacements (e.g., 15-20 $\mu \mathrm{m}$ ), phase-locked discharges followed with a remarkable temporal precision the enhanced resonance of the probe induced by higher command voltages (e.g., $\sim 1 \mathrm{kHz}$ ) (Fig. 6). It should be noted that, in traces and PSTHs shown in Figures 5 and 6, conduction times between the follicles and recording sites plus the response time lag of the stimulator have been subtracted from the records. Thus, the timing of discharges corresponds to the actual encoding points during the displacement curves.

The frequency-following ability of PR5 cells was still remarkably high, but often demonstrated dependency on stimulus parameters. Figure 7 shows a representative example of the frequency-following ability of a tonic PR5 unit that discharged phase-locked spike doublets (or triplets) at 50 and $100 \mathrm{~Hz}$, and single action potentials at stimulus frequencies of 200 and 350 $\mathrm{Hz}$. To compare the frequency-following ability of PR5 cells, a modulation index was computed for cells that always responded to the initial stimulus cycle of a 10 cycle sequence (as shown in Fig. 7) that was presented at various frequencies. This index consisted of the proportion of subsequent stimulus cycles (2-10) that generated at least one spike. The graphs of Figure 8 are representative of the various profiles of frequency modulation observed in a population of 25 cells (19 single-whisker and 6 multiwhisker units) in which the test was performed. Cell in Figure $8 \mathrm{~A}$ still responded $70 \%$ of the time to $400 \mathrm{~Hz}$ whisker deflections but failed to follow electrical stimulation of the follicle at the same frequency (asterisks indicate stimulus artifacts in the inserted PSTH). The two simultaneously recorded units in Figure $8 \mathrm{~B}$ and 


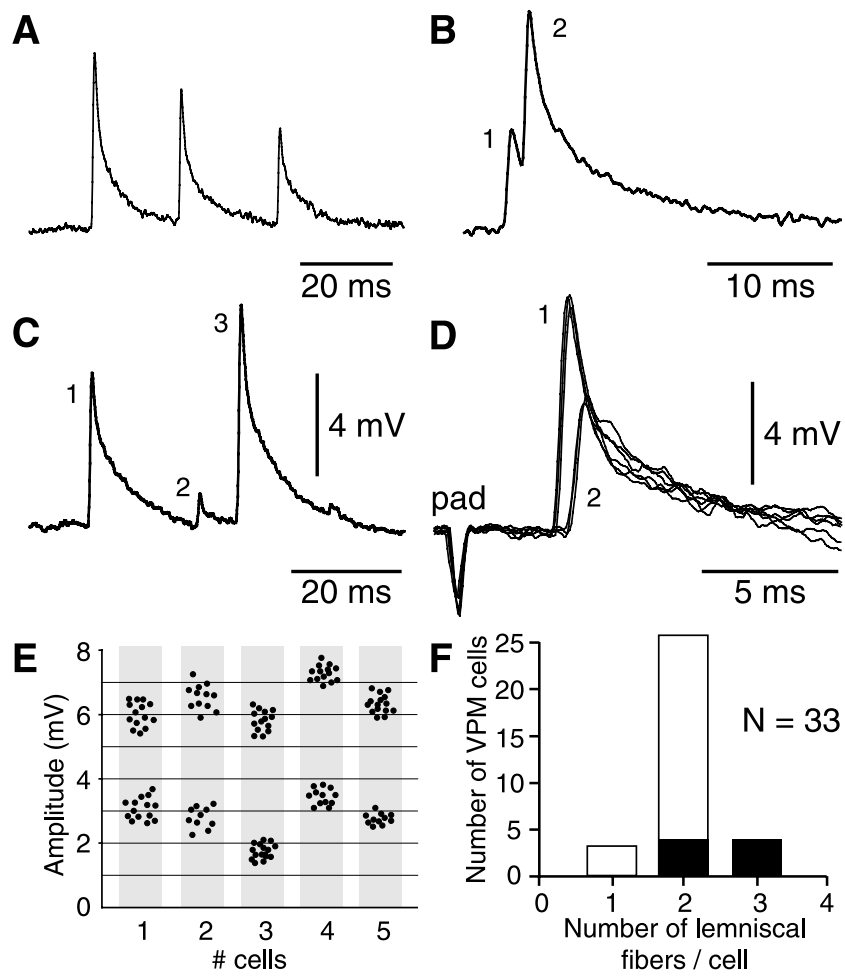

Figure 4. Criteria used to estimate the number of lemniscal fibers that contact single barreloid cells. A, Trace shows the synaptic depression observed when whisker-evoked EPSPs occurred at short intervals. In this case, it was inferred that the EPSP sequence resulted from the firing of a single presynaptic fiber. $B, C$, Traces show cases that break the rule of synaptic depression. To generate the sequences shown in $B$ and $C$, two and three presynaptic fibers are required, respectively. $D$, Additional evidence for the convergence of lemniscal fibers on the same barreloid cells was obtained after electrical stimulation of the follicle (pad) or of the medial lemniscus. Superimposed traces $(n=6)$ show single-fiber EPSPs of different amplitudes evoked at slightly different stimulus intensities. E, Scatter plots summarize the amplitude distribution of evoked EPSPs in five additional cells (each plot contains $20-30$ measures). $F$, 0 n the basis of these criteria, the histogram shows the number of lemniscal fibers that contact each of the 33 barreloid cells analyzed. Filled bars represent cells that were strongly driven by multiple whiskers, and open bars represent single-whisker units.

the separately recorded units in $C$ and $D$ show different profiles of frequency modulation. For the unit in Figure $8 E$, the capacity to respond to high-frequency deflections was direction sensitive, whereas, for the unit in $F$, the percentage of modulation was modified by changing deflection amplitude. With the latter unit, at $150 \mathrm{~Hz}$, the percentage of modulation increased from 76 to $92 \%$ as deflection amplitude was reduced to $32 \mu \mathrm{m}$, but decreased to $32 \%$ for displacements of $83 \mu \mathrm{m}$. Together, these results reveal that, although trigeminoprincipalis synapses can transfer high rates of whisker deflection, stimulus-dependent response transformations occur in the PR5. The actual frequencyfollowing ability of a particular cell might depend on intranuclear synaptic actions in which the response properties of costimulated primary afferents play a crucial role (see Discussion).

At first sight, the patterns of EPSPs recorded in barreloid cells during high-frequency whisker deflections closely resemble those of spike discharges in PR5 units. Subtle differences are present, however, all being related to the phase relationship of the EPSPs. In many units, like that shown in Figure $9 A-C$, EPSPs were triggered during both the rising and falling phases of the first stimulus half-cycle, but only those associated with the falling phase persisted during the following cycles. In another cell (Fig. 9D-F), EPSPs occurred during both downward and upward whisker dis- placements at $20 \mathrm{~Hz}$, but the responses to downward deflections were completely obliterated at higher frequencies. Such transformations likely involve a frequency-dependent discharge drop out among the lemniscal fibers that impact on individual cells.

By far, the gating of spike discharges was the most obvious feature observed in the thalamus. Local inhibitory mechanisms apparently play little role in spike suppression except perhaps for the first cycles after stimulus onset. Suppression was not produced by a state-dependent mode of burst discharges and rhythmic IPSPs but seems principally related to synaptic depression. Thus, if the remarkable frequency-encoding ability of prethalamic cells is to be used by the cortex, additional mechanisms that promote spike triggering will be required to overcome depression (see Discussion).

\section{Instantaneous firing rates in PR5 and barreloid cells}

Like primary afferent axons, PR5 cells can fire action potentials at very short intervals. Bursts of two to four actions potentials with intraburst frequencies $>1 \mathrm{kHz}$ (maximal frequency, $\sim 1.3 \mathrm{kHz}$ ) were commonly observed at stimulus onset (Fig. 10). These high firing rates were clearly related to the stimulus parameters, because, in most cells, the interspike interval was shortened progressively as deflection velocity or amplitude was increased. However, this relationship was not further analyzed in the present study because of the limited range of displacement offered by the stimulator (but see Shipley, 1974). Interestingly, PR5 bursts induced corresponding clusters of lemniscal EPSPs (Fig. $10 B$ ) that could, in turn, produce high-frequency burst discharges in barreloid cells (Fig. 10C; see also Fig. 9A-C). Interspike intervals within the synaptically driven bursts were as short as in bursts intrinsically driven by the activation of the low-threshold $\mathrm{Ca}^{2+}$ spike (Fig. 10C, compare traces recorded without and with hyperpolarizing current). The highest firing rate observed in synaptically driven bursts was $\sim 700 \mathrm{~Hz}$.

\section{Additional observations}

In a previous study (Brecht and Sakmann, 2002), it was reported that a small proportion of VPM cells were solely inhibited by principal whisker deflection. Cases of early inhibition were also observed in our experiments $(n=4)$, but an initial excitatory response could always be elicited by changing the parameters of stimulation, especially the direction of displacement. In the same study, it was also reported that sensory-evoked EPSPs in multiwhisker-responsive units exhibited longer rise times than those observed in relay cells dominated by a single whisker. Whisker-evoked EPSPs with rise times of 2-3 msec were indeed recorded in two multiwhisker cells, but these two units were situated deep in the ventral lateral part of the VPM (below A1responsive cells), a region principally innervated by the interpolaris division of the trigeminal nucleus (Pierret et al., 2000). These units were not included in our analysis protocol.

\section{Discussion}

In the present study, we investigated some operational features of lemniscal synapses in thalamic barreloids and the ability of the whisker-to-barreloid pathway to relay high-frequency sensory inputs. Results show that vibrissa information is conveyed with a high degree of synaptic security to individual barreloid cells by a small number of lemniscal axons, and that cells forming the whisker-to-barreloid pathway can follow in a one-to-one, phaselocked manner high-frequency whisker deflections. 


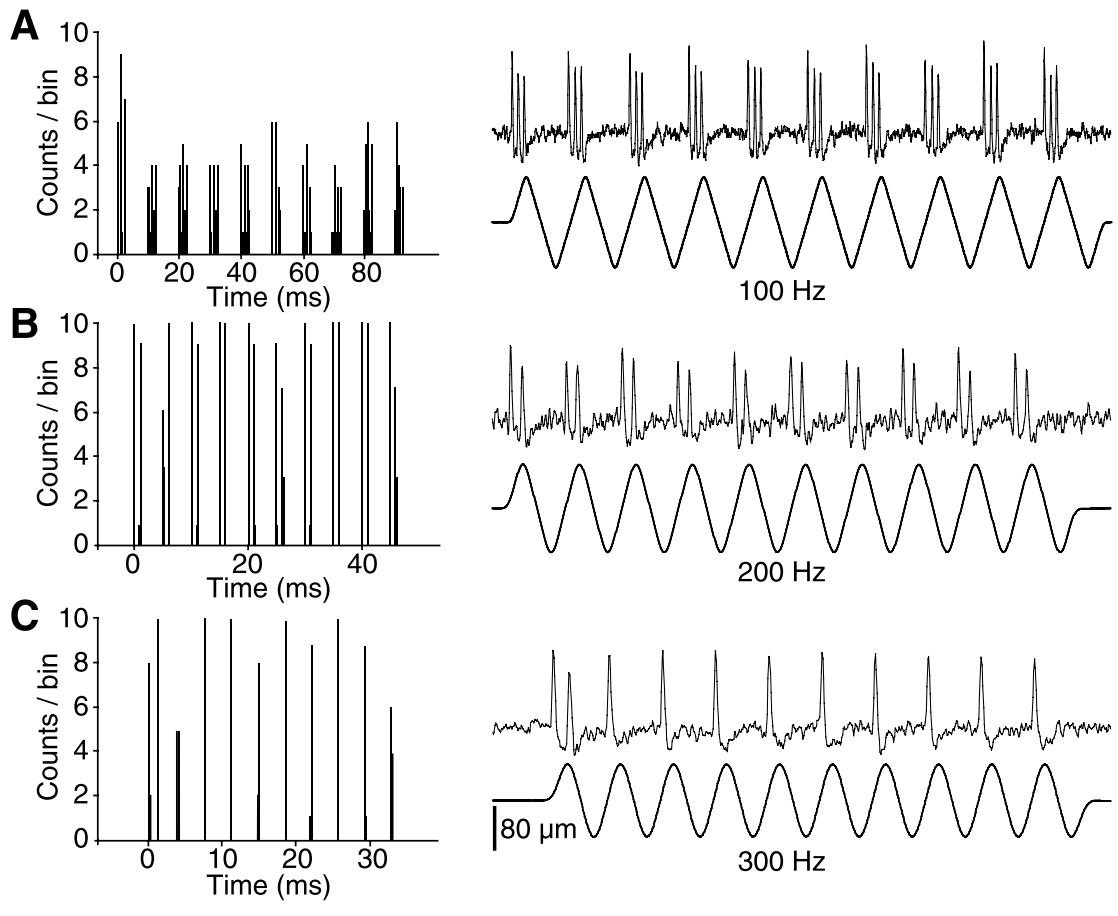

Figure 5. Frequency-following property of primary vibrissa afferents. The left-hand panels show PSTHs of cell responses to the stimulus sequences displayed in the right-hand panels. Each PSTH compiles 10 sequences.

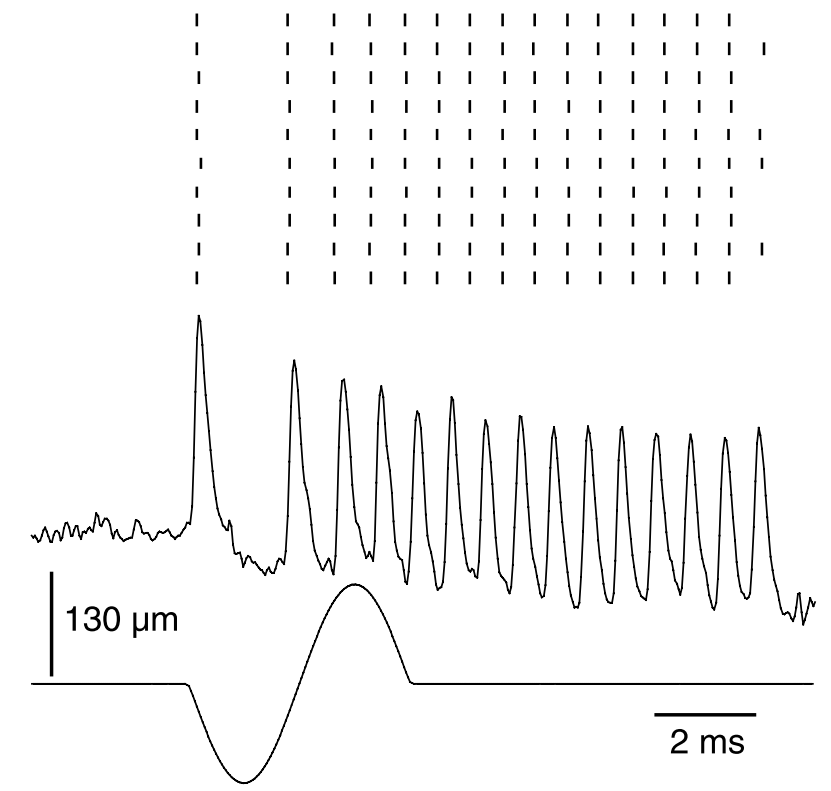

Figure 6. Encoding of high-frequency whisker deflections by primary afferent axons. This B2-responsive unit fired in a one-to-one manner (middle trace) in phase with the resonance frequency of the stimulator (e.g., $\sim 1 \mathrm{kHz}$ ) (Fig. 1). Note the remarkable time locking of the discharges in the raster display.

\section{Convergence of PR5 axons on barreloid cells}

The axonal field of PR5 cells in thalamic barreloids is small (e.g., $\sim 80 \mu \mathrm{m}$ ) (Veinante and Deschênes, 1999) and contains 25-60 boutons that form large terminals with multiple, closely spaced synaptic contacts on the proximal dendrites of relay cells (Spacek and Lieberman, 1974; Williams et al., 1994). These factors combine to produce a fine-grained map of vibrissa inputs and a high degree of synaptic security of transmission through the barre- loids. The small size of lemniscal axon terminal fields and the selective clustering of terminations on proximal dendrites thus impose a constraint to the number of axons that can impact on a relay cell. Assuming that no spike failure occurs at axonal branch points, and that all of the lemniscal synapses depress upon repetitive activation, our results indicate that at least one to three PR5 axons are required to produce the patterns of unitary EPSPs observed in single cells after whisker deflection. It should be emphasized that the overshooting criterion used to infer the number of presynaptic fibers may lead to an underestimate of synaptic convergence if two (or more) fibers produce EPSPs of approximately equal amplitude. In the present study, the degree of convergence estimated from that type of analysis was also supported by electrical stimulation of the whisker follicles, which adds reliability to the analysis protocol. A small degree of convergence was also found in cells that robustly responded to multiple whiskers, indicating that the synthesis of multiwhisker receptive fields relies on patterns of axonal convergence within the PR5 itself (for additional evidence, see Varga et al., 2002; Minnery and Simons, 2003). Because all of the axons that converge on a cell produce EPSPs of similar rise times, but of different amplitudes, one can conclude that size differences are likely related to the number of contacts each fiber establishes with the relay cell proximal dendrites.

The small number of lemniscal axons per relay cell is also suggested when one compares the pattern of unitary EPSPs in VPM cells with that of cell discharges in the PR5. In both cases, an increase in stimulus frequency results in simplified response patterns, which eventually consist of one spike and one single-fiber EPSP per cycle in PR5 and VPM cells, respectively. It was not possible, however, to identify within a complex EPSP sequence which of them resulted from the firing of the same presynaptic axon. Because the response properties of each converging axon could not be identified, the rules that govern convergence on barreloid cells remain undetermined. A reasonable assumption would be that input selection operates through the direction selectivity of incoming fibers, because this receptive-field property seems well conserved in the whisker-to-barreloid pathway ( $\mathrm{Si}$ mons and Carvell, 1989; Hartings et al., 2000; Minnery and Simons, 2003).

\section{Frequency-following ability in the} whisker-to-barreloid pathway

On the basis of previous results obtained in cats (Gottschaldt and Vahle-Hinz, 1981), it came as no surprise that primary vibrissa afferents in rats could encode in a one-to-one manner $1 \mathrm{kHz}$ whisker vibrations. However, it was surprising to find that signals generated by very high rates of whisker deflection were faithfully relayed across two central synapses up to the thalamus. One-toone, phase-locked discharge to stimulus rates of $>200 \mathrm{~Hz}$ were commonly observed in the PR5, and, in some cells, testing the limit exceeded the performance of the stimulator. Such a high degree of reliability and temporal precision is what would be expected for an active system to discriminate fine-grained textured surfaces. 
Because individual primary vibrissa afferents distribute multiple clusters of terminations in topographically related barrelettes, single PR5 cells should receive convergent inputs from a number of sensory axons (Hayashi, 1980; Jacquin et al., 1993). In addition, intersubnuclear projections and both presynaptic and postsynaptic inhibitory mechanisms should contribute to response transformations in the nucleus (Jacquin et al., 1990; Bae et al., 2000). Our results suggest transformations that involve inputs from primary afferents with different amplitude and/or velocity thresholds. For instance, the rise in frequency modulation that was observed as stimulus amplitude was decreased suggests a mechanism of input-output selection whereby the activation of higher-threshold afferents shuts off the relay of lower-threshold messages. Indeed, in the absence of inhibition, one could hardly explain how an increase in deflection amplitude, and hence in the size of the afferent volley, could depress synaptic transmission. A mechanism of intranuclear inhibition is also suggested by the fact that similar rates of suprathreshold whisker deflection and follicular stimulation can produce different entrainment patterns. The directional sensitivity of the modulation indexes gives another indication of input transformations in the PR5, although, in this case, it is not clear how much of this could occur at the level of primary afferents. Thus, for the moment, additional studies will be required to determine the relative importance of the various stimulus parameters in shaping the output of PR5 cells.

In contrast with PR5 cells, barreloid neurons exhibited a more limited frequency range of entrainment. Substantial differences in the frequency following of thalamic prepotentials and somal spikes have also been reported by Gottschaldt et al. (1983). Synaptic depression primarily contributes in filtering the output of relay cells, and this mechanism increases in efficacy with frequency. Thus, if the remarkable frequency-following ability of prethalamic cells is to be used by the cortex, presynaptic and/or postsynaptic facilitatory mechanisms should operate to overcome depression. A facilitatory action on whiskerevoked responses has been demonstrated previously in barreloid cells after stimulation of the brainstem cholinergic system (Castro-Alamancos, 2002b), but it is unclear to what extent the enhancement of transmission also involved the participation of other inputs (e.g., corticothalamic cells) that were coactivated by high-frequency brainstem stimulation. Another intriguing possibility is that, in behaving rats, the whisking behavior might represent a motor strategy that counteracts depression. As rats whisk across object surfaces, they can change the amplitude, velocity, and direction of motion (Sachdev et al., 2002), which produces ever-changing patterns of lemniscal inputs in different sets of fibers during the scan of regularly textured surfaces. Therefore, the relay of sensory inputs of high spatial frequency may not actually rely on the frequency-following ability of individual thalamic neurons but rather on phase-locked discharges among an ensemble of relay cells having different response preferences.

\section{Burst discharges in the whisker-to-barrel pathway}

Principalis cells demonstrated a remarkable capacity to discharge at high frequency; bursts of two to four spikes emitted at $>1 \mathrm{kHz}$ were commonly observed at stimulus onset. These initial bursts often resulted in the triggering of thalamocortical spike bursts of lower frequency (e.g., 300-600 Hz). In recent studies, a case was made for the potent drive thalamic bursts exert on cortical neurons, especially when they occur after silent periods of $>100 \mathrm{msec}$ (Sherman, 2001; Swadlow and Gusev, 2001). Hence, it was proposed that deinactivation of the low-threshold conductance during the waking state could participate in sensory processes by generating spike bursts that serve to overcome depression at cortical synapses. This proposal also implies that reticular thalamic cells fire in bursts to generate IPSPs large enough for deinactivating the low-threshold spike in relay cells, with the risk of inducing dampened oscillations in the system. The present results suggest that high-frequency sensory-driven bursts might do the same job in a safer way. If, as suggested by the recording of local field potentials in the barreloids (Temereanca and Simons, 2003), thalamic cells can be induced to fire in synchrony by whisker deflection, then sensory-driven bursts should potently contribute to enhance cortical responsiveness.

The synaptic relay of vibrissal inputs in the VPM is reminiscent of that of retinal inputs in the lateral geniculate nucleus. A number of studies have shown that retinal EPSPs have fast rise times, exhibit an all-or-none unitary behavior, and depress at short intervals (Eysel, 1976; Crunelli et al., 1987; Bloomfield and Sherman, 1988; Turner and Salt, 1998). The limited convergence ratio in the trigeminothalamic circuitry is also found in the visual system in which most geniculate cells receive the bulk of their 
A

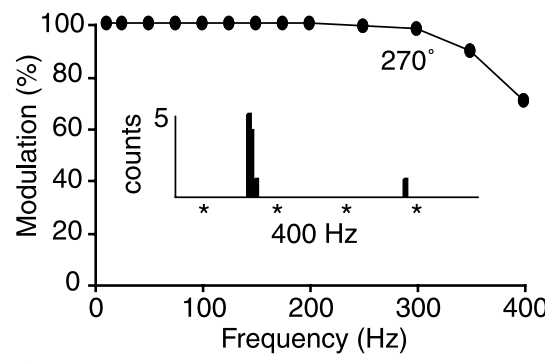

C

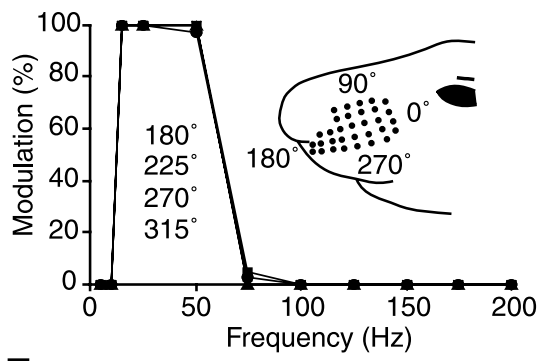

E

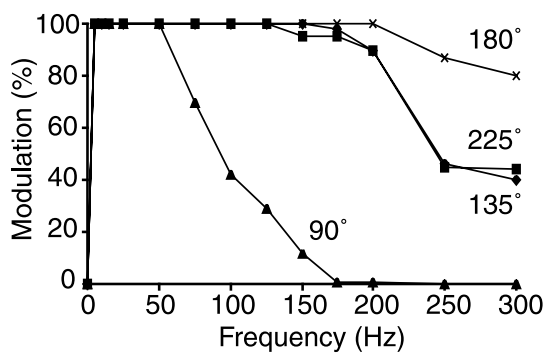

B

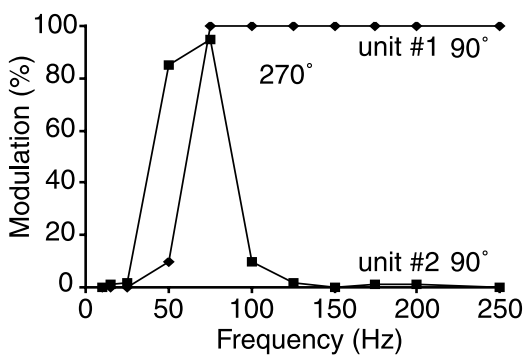

D

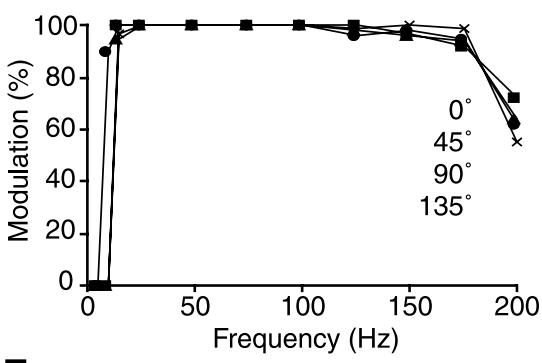

$\mathbf{F}$

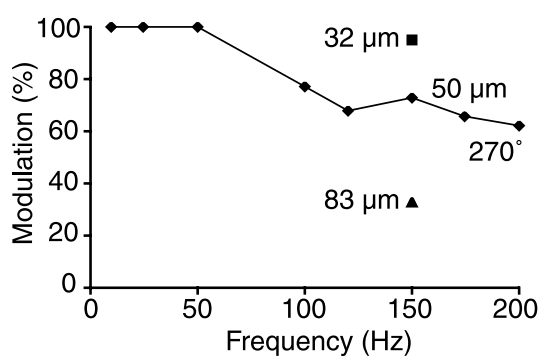

Figure 8. Representative examples of the ability of PR5 cells to follow high-frequency whisker stimulation. Cells were driven by bursts of 10 cycle sinusoidal displacements in different directions $\left(0^{\circ}\right.$, caudalward; $90^{\circ}$, upward, etc.) as shown in the inset in C. All of the units responded with at least one spike to the first cycle of each stimulus sequence. The percentage of modulation was computed by averaging the number of times the unit responded with at least one spike to each of the subsequent 9 cycles across a series of 10 stimulus sequences. The inserted PSTH in A shows responses evoked by electrical stimulation of the follicle (asterisks, 4 shocks at $400 \mathrm{~Hz}$ ). See Results for full description.

retinal inputs from a small number of axons (two to six) (Cleland et al., 1971; Hamos et al., 1987; Mastronarde, 1992). These common features suggest that both systems may use similar coding strategies in which the timing and synchrony of discharges play a crucial role (Alonso et al., 1996; Usrey et al., 1998).

\section{References}

Ahissar E, Sosnik R, Haidarliu S (2000) Transformation from temporal to rate coding in a somatosensory thalamocortical pathway. Nature 406:302-306.

Alonso JM, Usrey WM, Reid RC (1996) Precisely correlated firing in cells of the lateral geniculate nucleus. Nature 383:815-819.

Bae YC, Ihn HJ, Park MJ, Otterson OP, Moritani M, Yoshida A, Yoshio S (2000) Identification of signal substances in synapses made between primary afferents and their associated axon terminals in the rat trigeminal sensory nuclei. J Comp Neurol 418:299-309.

Bloomfield SA, Sherman MS (1988) Postsynaptic potentials recorded in neurons of the cat's lateral geniculate nucleus following electrical stimulation of the optic chiasm. J Neurophysiol 60:1924-1945.

Brecht M, Sakmann B (2002) Whisker maps of neuronal subclasses of the rat ventral posterior medial thalamus, identified by whole-cell voltage recording and morphological reconstruction. J Physiol (Lond) 538:495-515.

Carvell GE, Simons DJ (1990) Biometric analyses of vibrissal tactile discrimination in the rat. J Neurosci 10:2638-2648.
Castro-Alamancos MA (2002a) Properties of primary sensory (lemniscal) synapses in the ventrobasal thalamus and the relay of high-frequency sensory inputs. J Neurophysiol 87:946-953.

Castro-Alamancos MA (2002b) Different temporal processing of sensory inputs in the rat thalamus during quiescent and information processing states in vivo. J Physiol (Lond) 539:567-578.

Cleland BG, Dubin MW, Levick WR (1971) Sustained and transient neurones in the cat's retina and lateral geniculate nucleus. J Physiol (Lond) 217:473-496.

Crunelli V, Kelly JS, Leresche N, Pirchio M (1987) On the excitatory postsynaptic potentials evoked by stimulation of the optic tract in the rat lateral geniculate nucleus. J Physiol (Lond) 384:603-618.

Eysel UTH (1976) Quantitative studies of intracellular potentials in the lateral geniculate nucleus of the cat with respect to optic tract stimulus response latencies. Exp Brain Res 25:469-486.

Friedberg MH, Lee SM, Ebner FF (1999) Modulation of receptive field properties of thalamic somatosensory neurons by the depth of anesthesia. J Neurophysiol 81:2243-2252.

Gibson JM, Welker WI (1983a) Quantitative studies of stimulus coding in first-order vibrissa afferents of rats. 1. Receptive field properties and threshold distributions. Somatosens Res 1:51-67.

Gibson JM, Welker WI (1983b) Quantitative studies of stimulus coding in first-order vibrissa afferents of rats. 2. Adaptation and coding of stimulus parameters. Somatosens Res 1:95-117.

Gottschaldt K-M, Vahle-Hinz C (1981) Merkel cell receptors: structure and transducer function. Science 214:183-186.

Gottschaldt K-M, Vahle-Hinz C, Hicks TP (1983) Electrophysiological and micropharmacological studies on mechanisms of input-output transformation in single neurones of the somatosensory thalamus. In: Somatosensory integration in the thalamus (Macchi G, Rustioni A, Spreafico R, eds), pp 199216. Amsterdam: Elsevier.

Guic-Robles E, Valdivieso C, Guajardo G (1989) Rats can learn a roughness discrimination using only their vibrissal system. Behav Brain Res 31:285-289.

Hamos JE, Van Horn SC, Raczkowski D, Sherman SM (1987) Synaptic circuits involving an individual retinogeniculate axon in the cat. J Comp Neurol 259:165-192.

Hartings JA, Simons DJ (1998) Thalamic relay of afferent responses to 1- to $12-\mathrm{Hz}$ whisker stimulation in the rat. J Neurophysiol 80:1016-1029.

Hartings JA, Temereanca S, Simons DJ (2000) High responsiveness and direction sensitivity of neurons in the rat thalamic reticular nucleus to vibrissa deflections. J Neurophysiol 83:2791-2801.

Hayashi H (1980) Distribution of vibrissae afferent fiber collaterals in the trigeminal nuclei as revealed by intra-axonal injection of horseradish peroxidase. Brain Res 183:442-446.

Jacquin MF, Chiaia NL, Haring JH, Rhoades RW (1990) Intersubnuclear connections within the rat trigeminal brainstem complex. Somatosens Mot Res 7:399-420.

Jacquin MF, Renehan WE, Rhoades RW, Panneton WM (1993) Morphology and topography of identified primary afferents in trigeminal subnuclei principalis and oralis. J Neurophysiol 70:1911-1936.

Lichtenstein SH, Carvell CA, Simons DJ (1990) Responses of rat trigeminal ganglion neurons to movements of vibrissae in different directions. Somatosens Mot Res 7:47-75.

Mastronarde DN (1992) Nonlagged relay cells and interneurons in the cat lateral geniculate nucleus: receptive-field properties and retinal inputs. Vis Neurosci 8:407-441.

Miller KD, Pinto DJ, Simons DJ (2001) Processing in layer 4 of the neocortical circuit: new insights from visual and somatosensory cortex. Curr Opin Neurobiol 11:488-497.

Minnery BS, Simons DJ (2003) Response properties of whisker-associated 
trigeminothalamic neurons in rat nucleus principalis. J Neurophysiol 89:40-56.

Paxinos G, Watson C (1986) The rat brain in stereotaxic coordinates, Ed 2. Sydney: Academic.

Pierret T, Lavallée P, Deschênes M (2000) Parallel streams for the relay of vibrissal information through thalamic barreloids. J Neurosci 20:7455-7462.

Pinault D, Deschênes M (1992) The origin of rhythmic fast subthreshold depolarizations in thalamic relay cells of rats under urethane aneasthesia. Brain Res 595:295-300.

Pinto DJ, Hartings JA, Brumberg JC, Simons DJ (2003) Cortical damping: analysis of thalamocortical response transformations in rodent barrel cortex. Cereb Cortex 13:33-44.

Sachdev RNS, Takashi S, Ebner FF (2002) Divergent movement of adjacent whiskers. J Neurophysiol 87:1440-1448.

Salt TE (1987) Excitatory amino acid receptors and synaptic transmission in the rat ventrobasal thalamus. J Physiol (Lond) 391:499-510.

Sherman MS (2001) A wake-up call from the thalamus. Nat Neurosci 4:344-346.

Shipley MT (1974) Response characteristics of single units in the rat's trigeminal nuclei to vibrissa displacements. J Neurophysiol 37:73-90.

Shoykhet M, Doherty D, Simons DJ (2000) Coding of deflection velocity and amplitude by whisker primary afferent neurons: implications for higher level processing. Somatosens Mot Res 17:171-180.

Simons DJ, Carvell GE (1989) Thalamocortical response transformation in the rat vibrissa-barrel system. J Neurophysiol 61:311-330.

Sosnik R, Haidarliu S, Ahissar E (2001) Temporal frequency of whisker movement. I. Representations in brain stem and thalamus. J Neurophysiol 86:339-353.

Spacek J, Lieberman AR (1974) Ultrastructure and three-dimensional organization of glomeruli in rat somatosensory thalamus. J Anat 117:487-516.

Swadlow HA, Gusev A (2001) The impact of "bursting" thalamic impulses at a neocortical synapse. Nat Neurosci 4:402-408.

Temereanca S, Simons DJ (2003) Local field potentials and the encoding of whisker deflections by population firing synchrony in thalamic barreloids. J Neurophysiol 89:2137-2145.

Turner JP, Salt TE (1998) Characterization of sensory and corticothalamic excitatory inputs to rat thalamocortical neurons in vitro. J Physiol (Lond) 510:829-843.

Usrey WM, Reppas JB, Reid RC (1998) Paired-spike interactions and synaptic efficacy of retinal inputs to the thalamus. Nature 395:384-387.

Varga C, Sik A, Lavallée P, Deschênes M (2002) Dendroarchitecture of relay
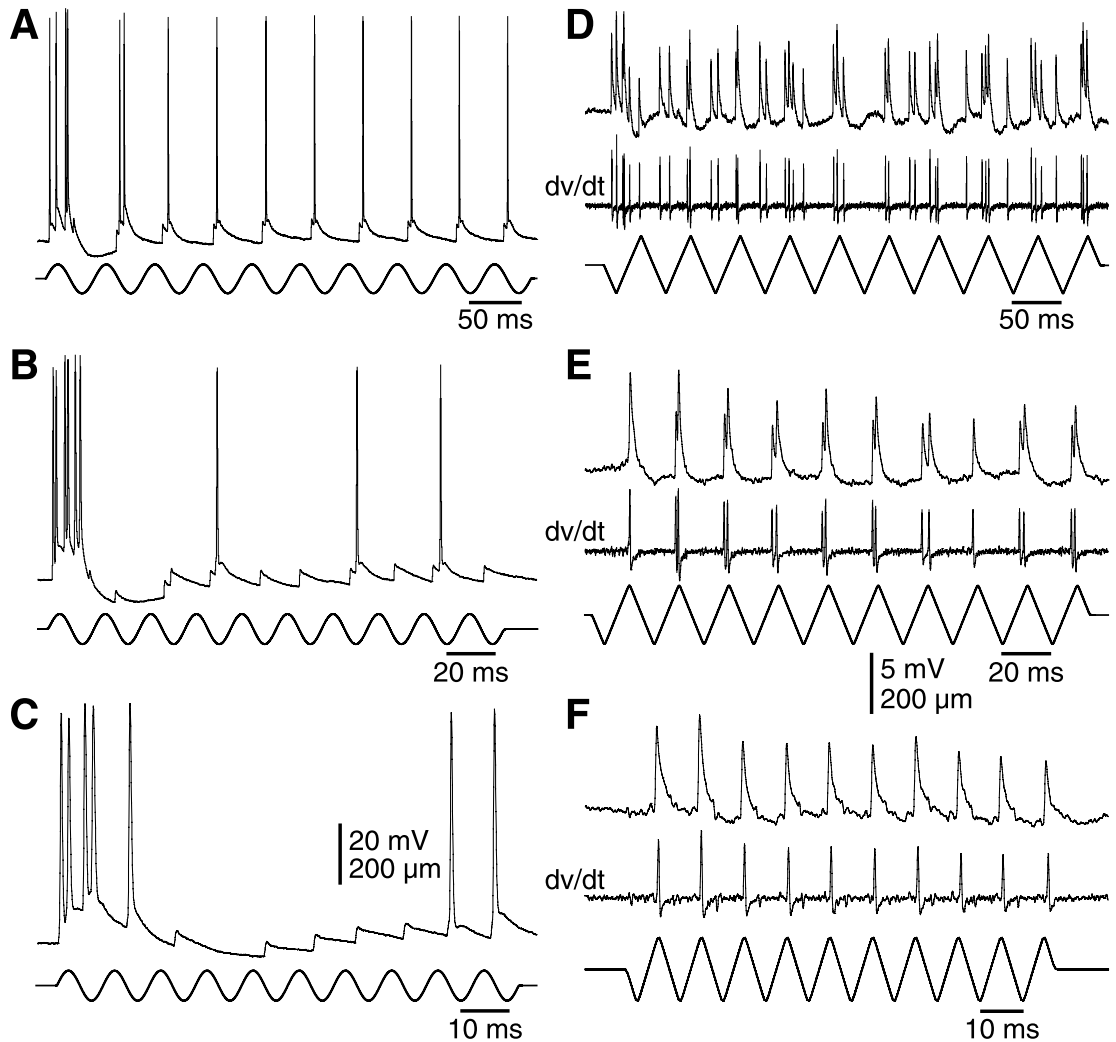

Figure 9. Patterns of lemniscal EPSPs evoked in barreloid cells by repetitive whisker deflections. $A-C$, Traces show responses of a (2-responsive barreloid cell to bursts of sinusoidal deflections at 20,50 , and $100 \mathrm{~Hz}$, respectively. $D-F$, Traces show EPSPs and their time derivatives recorded in another cell driven by bursts of triangular deflections. The cell was kept hyperpolarized to prevent spike discharges. Note that, at $20 \mathrm{~Hz}$ (D), EPSPs were evoked during both phases of the stimulus sequence (here, downward and upward deflections of vibrissa (4), but that, at higher rates of stimulation (50 and $100 \mathrm{~Hz}$ in $E$ and $F$, respectively), EPSPs only followed upward deflections.

cells in thalamic barreloids: a substrate for cross-whisker modulation. J Neurosci 22:6186-6194.

Veinante P, Deschênes M (1999) Single- and multi-whisker channels in the ascending projections from the principal trigeminal nucleus in the rat. J Neurosci 19:5085-5095.

Williams MN, Zahm DS, Jacquin MF (1994) Differential foci and synaptic organization of the principal and spinal trigeminal projections to the thalamus in the rat. Eur J Neurosci 6:429-453.

Zucker E, Welker WI (1969) Coding of somatic sensory input by vibrissae neurons in the rat's trigeminal ganglion. Brain Res 12:138-156. 
A
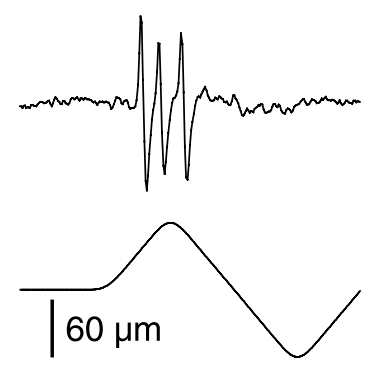

$5 \mathrm{~ms}$
PR5

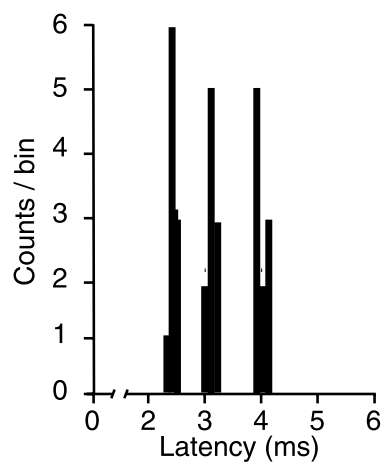

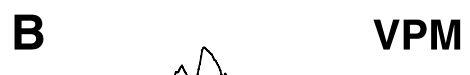
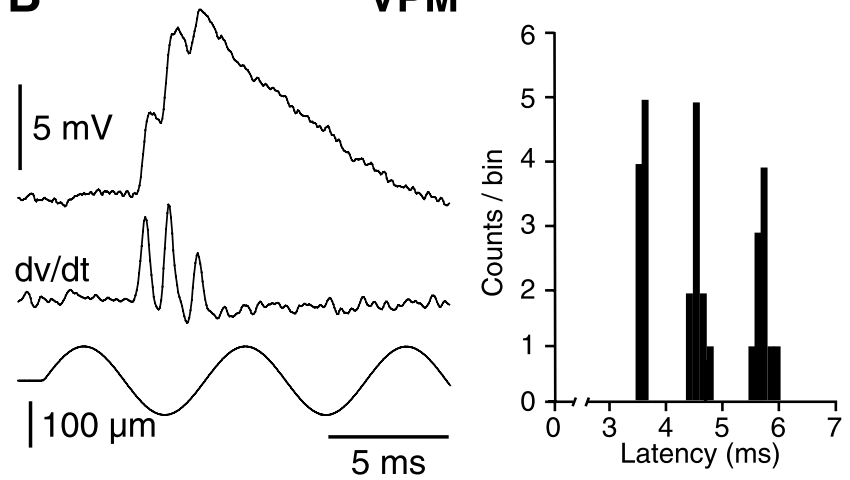

\section{C}

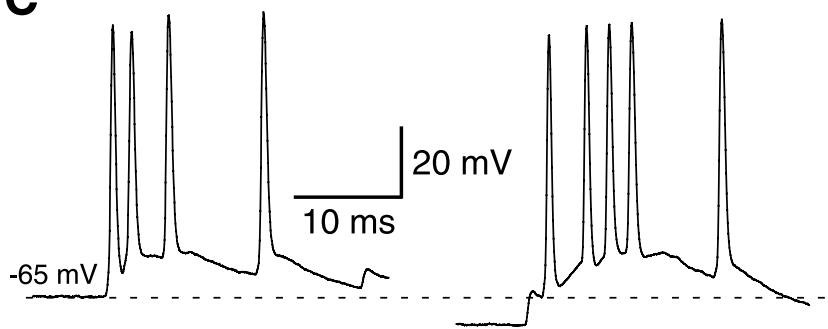

Figure 10. High firing rates induced in PR5 and barreloid cells by whisker deflections. $A_{t}$ Most PR5 units could generate stereotyped bursts of action potentials (intraburst frequencies, $>1 \mathrm{kHz}$ ) in response to high-velocity whisker displacements. $B$, In barreloid cells, PR5 bursts induced compound EPSPs with similar intraburst frequencies. $A, B$, PSTHs show spikes $(A)$ and EPSP counts ( $B$ ) (discriminated by the time derivative of EPSPS) evoked by the first deflections of 10 stimulus sequences. C, The left-hand trace shows a burst driven by lemniscal EPSPs in response to the first cycle of a $100 \mathrm{~Hz}$ stimulus sequence. The right-hand trace shows the response to the same stimulus during membrane hyperpolarization. The dotted line indicates the resting potential $(-65 \mathrm{mV})$. 